\title{
COMPORTAMIENTO ANTISOCIAL EN LAS ORGANIZACIONES: UNA REVISIÓN Y ANÁLISIS CONCEPTUAL
}

\author{
Julio César Álvarez Escalante, Francisco Javier Ruvalcaba Coyaso, \\ Bélgica María Romero de Loera y José Carlos Palacios Montoya \\ Universidad Autónoma de Aguascalientes \\ México
}

\begin{abstract}
RESUMEN
Considerando la diversidad conceptual para el estudio de los comportamientos antisociales dentro de las organizaciones se desarrolla este texto, una revisión y análisis teórico. A partir del panorama conceptual mostrado se hacen algunas críticas acerca de la tendencia a ser estudiado bajo el nombre de comportamientos contraproducentes en el trabajo y comportamiento desviado en el trabajo y sus implicaciones respecto a una visión que divide a la organización y a sus miembros o trabajadores, en donde destaca la dirección en que ocurren dichos comportamientos; trabajador hacia la organización, atribuida sobre todo a los rasgos de personalidad expuestos a determinadas condiciones laborales. Por último se plantean consideraciones para futuros trabajos en relación con los comportamientos antisociales en las organizaciones desde una perspectiva social, en las cuales se contemplen formas que no sólo consideren las cuestiones individuales de la problemática.
\end{abstract}

\section{Palabras Clave:}

psicología social, psicología del trabajo y las organizaciones, comportamiento antisocial en las organizaciones, comportamientos contraproducentes en el trabajo, comportamiento desviado en el trabajo

\section{ANTISOCIAL BEHAVIOR IN ORGANIZATIONS; A CONCEPTUAL ANALYSIS AND REVIEW.}

\begin{abstract}
Taking into account the existing conceptual diversity for the study of antisocial behaviors within organizations, the present text shows a review and theoretical analysis. From the conceptual panorama shown, some criticisms will be made about the tendency to be studied under the name of counterproductive behaviors at work and behavior sent at work and its implications regarding a vision that divides the organization and its members or workers, where the direction in which these behaviors occurs is marked; worker towards the organization, mainly attributed to specific personality traits to certain working conditions. Finally, considerations are raised for future works in relation to antisocial behaviors in organizations from a social perspective, in which ways are contemplated that do not only take into account the individual issues of the problem.
\end{abstract}

\section{Keywords:}

social phsycology, phsycology of works and organizations, antisocial behaviors in organizations, counterproductive workplace behavior, deviant workplace behavior

Bitácora del ARtículo:

| Recibido: 1 de Septiembre de 2020 | Aceptado: 07 enero de 2021 | Publicado en línea: Enero - Junio de 2021 | 


\title{
COMPORTAMIENTO ANTISOCIAL EN LAS ORGANIZACIONES: UNA REVISIÓN Y ANÁLISIS CONCEPTUAL
}

\author{
Julio César Álvarez Escalante, Francisco Javier Ruvalcaba Coyaso, \\ Bélgica María Romero de Loera y José Carlos Palacios Montoya \\ Universidad Autónoma de Aguascalientes \\ México
}

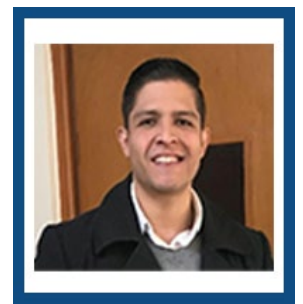

Julio César Álvarez Escalante UAA

Correo: cesar_alvarez08@hotmail.com

Lic. en Psicología y maestro en Investigación en Psicología por la Universidad Autónoma de Aguascalientes. Participó como profesor adjunto y en asistencia de investigación en problemáticas sobre psicología social. Actualmente Labora en la Secretaría de Salud del Estado de Aguascalientes. Es docente de la licenciatura en Psicología en la Universidad del Valle de México.

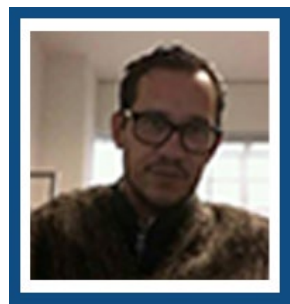

Francisco Javier Ruvalcaba Coyaso UAA

Correo: javier.ruvalcaba@edu.uaa.mx

Licenciado en Psicología, maestría en Psicología del Trabajo y las Organizaciones. doctorado en Psicología con énfasis en Psicología Social y Organizacional. Miembro del Sistema Nacional de Investigadores del 2014 al 2019. Coordinador de diplomados, curso y talleres sobre la norma 035 de riesgos psicosociales en el trabajo.

\section{CONTRIBUCIÓN DE LOS AutORES}

Julio César Álvarez Escalante concibió, y desarrollo el proyecto. | Francisco Javier Ruvalcaba Coyaso realizo revisiones en cuanto a redacción, estilo. Contribuciones en el análisis y conclusiones. I Bélgica María Romero de Loera realizo revisiones en cuanto a redacción, estilo, así como contribuciones en el análisis y conclusiones. |José Carlos Palacios Montoya realizo revisiones en cuanto a redacción, estilo.

\section{AGRADECIMIENTOS}

El artículo es parte de la tesis para optar al grado de maestría en Investigación en Psicología de la Universidad Autónoma de Aguascalientes, México, con apoyo de Conacyt.

\section{Datos de FiLiación de los Autores}

Julio César Álvarez Escalante, Departamento de Psicología de la Universidad Autónoma de Aguascalientes | Francisco Javier Ruvalcaba Coyaso, Departamento de Psicología de la Universidad Autónoma de Aguascalientes | Bélgica María Romero de Loera, Universidad Cuauhtémoc, campus Aguascalientes | José Carlos Palacios Montoya, Universidad Iberoamericana, campus Tijuana.

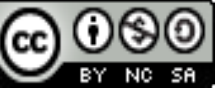

Copyright: (C) 2021 Álvarez-Escalante, J.C.; Ruvalcaba-Coyaso, F.J.; Romero de Loera, B.M; \& Palacios-Montoya, J.C.

Este es un artículo de acceso abierto distribuido bajo los términos de la licencia Creative Commons Reconocimiento-NoComercial 4.0 Internacional, por lo que su contenido gráfico y escrito se puede compartir, copiar y redistribuir total o parcialmente sin necesidad de permiso expreso de sus autoras con la única condición de que no se puede usar con fines directamente comerciales y los términos legales de cualquier trabajo derivado deben ser los mismos que se expresan en la presente declaración. La única condición es que se cite la fuente con referencia a la Revista Digital Internacional de Psicología y Ciencia Social y a sus autoras. 


\section{TABLA DE CONTENIDO}

CLASIFICACIONES DEL COMPORTAMIENTO ANTISOCIAL EN LAS ORGANIZACIONES

CONCEPTOS ALTERNATIVOS DEL COMPORTAMIENTO ANTISOCIAL ORGANIZACIONAL Y MANERAS DE ABORDAR EL PROBLEMA

ANÁLISIS CONCEPTUAL

CONCLUSIONES

REFERENCIAS 
$\mathbf{E}$ n el ámbito de la Psicología, el comportamiento antisocial y su prevención en los contextos organizacionales son temas recurrentes que han causado gran interés en los círculos académicos. Dichas problemáticas representan uno de los mayores obstáculos que enfrentan las organizaciones actuales en distintos países (Robinson y O'Leary-Kelly, 1998; Omar, Vaamonde y Uribe, 2012).

Autores como Appelbaum, laconi y Matousek (2014), Omar (2010) y Patlán, Navarrete y García (2010) exponen la manera en que los comportamientos antisociales en las organizaciones representan una problemática emergente que requiere mayor profundización para su comprensión debido a que tienen consecuencias para las organizaciones de manera significativa, pues abarca un amplio número de comportamientos negativos, como el robo, el consumo de sustancias, el ausentismo, hacer el trabajo de modo inapropiado, el acoso y hostigamiento, fraude, y agresiones físicas y verbales. De acuerdo con Appelbaum, Iaconi y Matousek (2014), los comportamientos antisociales pueden ser de tres maneras: 1) interpersonales, es decir, los que ocurren hacia otros miembros de la organización; 2) organizacionales, que se refieren a los que trasgreden las normas establecidas o que son dirigidos hacia los bienes de la organización, y 3) los anti-productivos, que son los que las personas efectúan para su propio provecho o distracción personal dentro de los horarios laborales.

Uno de los principales retos para el estudio de estas problemáticas es la diversidad de términos y clasificaciones utilizados para nombrarlos y explicarlos. Robinson y Bennett (1995) los denominan comportamientos desviados en el trabajo, Giacalone y Greenberg (1997) los definen comportamientos antisociales en las organizaciones, Sacket y De Vore (2001) los refieren como comportamientos contraproducentes en el trabajo, mientras que Vardi y Weitz (2003) los identifican como mala conducta en la organización. A diferencia de los autores citados, Griffin y O'Leary-Kelly (2004) utilizan un término más particular — "el lado obscuro del comportamiento organizacional" - para englobar los diversos comportamientos negativos en las organizaciones.

Así, los términos mencionados se han utilizado como sinónimos en distintas investigaciones (Griffin y López, 2005). Es importante subrayar que estos conceptos, dentro de sus definiciones, hacen referencia al mismo objeto de estudio o problema de las organizaciones. La multiplicidad de términos expuesta en líneas anterio- res puede generar confusiones para quienes pretenden acercarse a su estudio; por ejemplo, se puede pensar que se trata de diferentes problemáticas.

Debido a lo anterior, este texto tiene como objetivo hacer un recorrido por los diversos conceptos utilizados para estudiar los comportamientos antisociales dentro de las organizaciones. Se comienza por exponer las aportaciones de los autores que abonaron al estudio de los comportamientos antisociales en las organizaciones a partir de esta manera de referirlo. Después se muestran los distintos términos alternativos.

A partir de una revisión conceptual se reflejan algunas similitudes entre los términos con el objetivo de mostrar que abordan el mismo problema. En este sentido también se muestran las diferencias entre cada postura. Del mismo modo, se retoman las implicaciones de los estudios bajo la postura de comportamientos contraproducentes y comportamientos desviados en el lugar de trabajo, pues estas acciones efectuadas por el trabajador afectan los objetivos de la organización. De esta manera se visibiliza la división entre la organización y el trabajador. Desde esta perspectiva, enfocada en las características individuales de cada uno de sus miembros, el comportamiento antisocial es visto como las acciones negativas que comenten los sujetos en su lugar de trabajo y que tienen repercusiones en el desempeño laboral y la productividad de la organización.

Por último se propone, a modo de propuesta, comenzar a desarrollar formas de investigación e intervención para el estudio de los comportamientos antisociales en las organizaciones desde perspectivas sociales que logren englobar este fenómeno de manera integral.

\section{Comportamiento antisocial}

\section{EN LAS ORGANIZACIONES}

En el campo de la psicología, el estudio de los comportamientos antisociales no es una problemática reciente. En el ámbito organizacional se ha abordado desde hace ya algunos años sobre todo por la manera en que repercute en las organizaciones (Robinson y O'Leary-Kelly, 1998). Griffin y López (2005) mencionan, como una de las principales dificultades que se puede encontrar al estudiar esta problemática, la falta de consenso acerca de cómo definirla, generado una gran cantidad de términos, clasificaciones y hasta confusiones debido a la multiplicidad de formas para nombrarlos en el contexto de las organizaciones.

Por su parte, Giacalone y Greenberg (1997) hacen una de las primeras menciones de esta problemática en el contexto de las organizaciones. Definen el término "comportamiento antisocial en las organizaciones" 
como "los comportamientos dirigidos a causar daño o con intención de causarlo hacia los individuos, la organización, y de manera general a todas las personas involucradas dentro de ese contexto" (p. 7).

Giacalone y Greenberg (1997) sustentan que el interés central de la Psicología en el área organizacional es comprender las características individuales que tienen los sujetos que se involucran en conductas consideradas deshonestas. Así, se plantea como principal intervención la atención de los aspectos de personalidad que influyen en ellas. Del mismo modo, el comportamiento antisocial en las organizaciones es explicado mediante los aspectos de la personalidad y los factores del ambiente laboral que pueden llegar a ser un antecedente para generar este tipo de acciones. Argumentan también que el interés en conocer algunos factores relacionados con la ocurrencia de dichos comportamientos es algo que ya se ha visto en otras áreas de la Psicología que enfocan su atención en las acciones antisociales. Desde esta perspectiva organizacional, además de atender aspectos individuales o rasgos de personalidad, también se busca comprender los aspectos del clima y la cultura organizacional como desencadenantes o antecedentes de las acciones negativas en relación con las características del sujeto. En este sentido, estos comportamientos representan una oposición a los comportamientos considerados prosociales, los cuales buscan el bien y permiten el correcto desempeño de los individuos en su lugar de trabajo. En otras palabras, estas acciones son consideradas como algo "malo" o "negati$v^{\prime \prime}$ y "deshonesto" en la organización.

\section{Clasificaciones del comportamiento}

\section{ANTISOCIAL EN LAS ORGANIZACIONES}

A partir de la aportación de Giacalone y Greenberg (1997) se comienza a dar seguimiento al estudio de los comportamientos antisociales dentro del contexto organizacional. En este sentido, Robinson y O'Leary-Kelly (1998) continúan por la misma línea al usar el término "comportamiento antisocial en las organizaciones" para abarcar las acciones negativas que suceden de manera intencional y que van en contra de los principios de éstas, es decir, las acciones que ocurren hacia otros empleados o hacia la organización, y que tienen el potencial de producir daños físicos, económicos, psicológicos y emocionales.

Para Robinson y O'Leary-Kelly (1998), el término "comportamiento antisocial" representa una forma más completa de englobar esta problemática en comparación con otros términos que podrían utilizarse. Al igual que Giacalone y Greenberg (1997), el uso del término "antisocial" representa la oposición a los comporta- mientos prosociales, es decir, comportamientos buenos o favorables para las organizaciones.

Las aportaciones principales de Robinson y O'Leary-Kelly (1998) hacen referencia a la diversidad de acciones negativas que repercuten en las organizaciones: el ausentismo, el trabajo lento, daños a la propiedad, robo y acoso laboral y/o sexual. Dichas prácticas son perjudiciales para las organizaciones y para quienes las conforman; por lo regular son efectuadas con la intención de dañar o vulnerar a los otros.

De esta manera, es posible diferenciar las acciones hechas por los individuos en tres rubros: 1) las que repercuten de manera económica hacia la organización (como el daño dirigido a los bienes); 2) los comportamientos antisociales relacionados con los aspectos psicológicos o emocionales, como el acoso laboral y las agresiones físicas, y 3) las relacionadas con el desempeño inadecuado que afecta la productividad.

Por otra parte, Robinson y O'Leary-Kelly (1998) hablan acerca de los factores que pueden generar que se desencadenen estas acciones en las organizaciones. Explican que este fenómeno ha sido estudiado sobre todo como una cuestión individual. Destacan la necesidad de comenzar a considerar el contexto social del grupo para explicar la ocurrencia de estos comportamientos. Dichos autores describen la importancia del grupo de trabajo y su influencia para que cada uno de sus miembros comience a generar comportamientos antisociales.

Por otro lado, Griffin y O'Leary-Kelly (2004) proponen una manera distinta y particular de referirse a los comportamientos antisociales, porque los nombran como el "lado oscuro del comportamiento organizacional". Al hablar de lado oscuro del comportamiento organizacional dichos autores también hacen referencia a los aspectos antisociales en un contexto organizacional. Así, introducen una categorización de las acciones antisociales medible u objetivas y las subjetivas. Las primeras son las que pueden tener repercusión sobre todo económica o material en la organización, como el robo o el fraude, o dañar alguna propiedad de la misma. En cuanto a las segundas, las acciones antisociales subjetivas, mencionan que pueden ser el daño hacia la reputación de algún individuo, acciones de acoso u hostigamiento, agresiones físicas o de cualquier tipo de repercusión psicológica.

En cuanto a la primera categorización descrita en líneas anteriores, el principal interés está dirigido a los bienes de la organización, porque estos comportamientos antisociales son nombrados como algo que puede ser medible, es decir, los daños que pueden ser detectados con más facilidad. En cuanto a los otros comporta- 
mientos antisociales de manera subjetiva, están los que no son observables con facilidad.

De esta manera, en las aportaciones de Griffin y O'Leary-Kelly (2004) se comienza a observar esa distinción entre trabajador y organización la clasificación de comportamientos antisociales: 1) los comportamientos negativos que dañan o son perjudiciales al ser humano (incluyendo los comportamientos que dañan o son perjudiciales al trabajador y a otros miembros de la organización), y 2) los comportamientos negativos que dañan o son perjudiciales a la organización (incluyen los comportamientos que generan un costo a la organización y los que se producen y no generan costo alguno).

En esta clasificación se considera una diversidad de actos considerados antisociales: la alienación laboral, el síndrome de burnout, estrés laboral, evitación del trabajo, resistencia al cambio, ausentismo, violencia, acoso en el trabajo, sobrecarga laboral, diversas formas de agresión, dañar, destruir o robar el equipo y las instalaciones, el consumo de sustancias adictivas, trabajar con más lentitud siempre que sea posible y el no comunicar ideas favorables para la organización (Patlán, García y Navarrete, 2011).

Como se observa, los autores que se han retomado hasta el momento han utilizado el término de comportamientos antisociales en el contexto de las organizaciones. Es evidente que todos hacen referencia a un conjunto de actos emitidos de modo intencional por algún individuo y acciones que tienen la posibilidad de causar daño. Las acciones antisociales pueden llegar a tener consecuencias legales significativas, por lo que hay una gran diversidad de acciones que pueden clasificarse en esta categoría antisocial como las ya mencionadas. En las posturas propuestas por los autores que han abonado al estudio de este problema se refleja este punto en común: el notable interés en la comprensión de por qué ocurren las acciones antisociales y de las consecuencias que tienen para las organizaciones y sus miembros.

\section{Conceptos alternativos}

\section{DEL COMPORTAMIENTO ANTISOCIAL}

\section{ORGANIZACIONAL Y MANERAS DE ABORDAR}

\section{EL PROBLEMA}

El modo de nombrar los comportamientos antisociales en el contexto organizacional es algo complejo, porque ha tenido diferentes denominaciones a lo largo del tiempo, por lo que es necesario clarificar los términos utilizados hasta ese momento. Por otra parte, también es importante destacar algunas particularidades de cada uno de estos términos para clasificar las acciones antisociales, así como algunas aportaciones respecto a cómo han estudiado el fenómeno desde estas perspectivas, en donde han sido considerado los rasgos de personalidad, así como como la relación de los mismos con los aspectos del ambiente laboral como antecedente a estas acciones.

Robinson y Bennett (1995) pueden ser considerados los pioneros en el estudio de esta problemática, incluso antes de ser denominada como comportamientos antisociales en las organizaciones. Para dichos autores estas acciones se denominan comportamientos desviados en el trabajo y son definidos como "un comportamiento que de forma voluntaria viola significativamente las normas de una organización, y al hacerlo amenaza el bienestar de la organización, sus miembros, o de ambos" (p. 556). En esta visión, las normas de la organización son la pauta principal para establecer cuando algo es un comportamiento desviado o un comportamiento antisocial. A partir de esta definición los autores mencionados desarrollaron una de las primeras tipologías de comportamientos antisociales o desviados en el trabajo. Esta investigación permitió una primera clasificación de las manifestaciones antisociales o desviadas en las organizaciones. La manera de clasificar la desviación laboral por parte de estos autores es a partir de los comportamientos desviados de modo interpersonal y los que son hacia la organización. Dentro de estas dos clasificaciones, Robinson y Bennett (1995) proponen cuatro categorías: 1) el comportamiento desviado hacia la producción; 2) hacia la propiedad; 3) hacia las políticas de la organización, y 4) la agresión personal. En cada una de ellas es posible observar los ejemplos de las acciones que constituyen cada una de las categorías.

En relación con la producción, Robinson y Bennett (1995) plantean las siguientes acciones: cuando los trabajadores salen sin permiso antes de la hora establecida, toman descansos prolongados, trabajan de manera más lenta o el mal uso de los recursos del lugar de trabajo. En cuanto a las acciones relacionadas con la propiedad se encuentran el sabotear el equipo de trabajo, mentir acerca de las horas de trabajo y robar objetos del lugar de trabajo, por mencionar sólo algunas. Estas dos primeras categorías están relacionadas con los daños hacia la organización.

Por otra parte, Robinson y Bennett (1995) abordan los comportamientos desviados en relación con las políticas de la organización. Aquí se encuentran situaciones como la muestra de favoritismos, culpar a lo compañeros de trabajo, así como hacer comentarios negativos acerca de los compañeros. Por último están las agresiones personales, es decir, el acoso y hostigamiento, agresiones verbales, robar objetos de los compañeros o 
poner en peligro a los demás. Estas dos últimas categorías forman parte de las desviaciones interpersonales.

Robinson y Bennett (1995) argumentan que las desviaciones de agresiones personales y las de la propiedad son consideradas las más severas. Las desviaciones de las políticas y la producción son consideradas menos severas. Postura similar a la de Robinson y O'Leary-KeIly (1998), quienes también establecen una clasificación desde la cual estos comportamientos desviados o antisociales son de mayor gravedad.

La aportación de estos autores permitió una mejor y más detallada comprensión y categorización de cada una de estas maneras de actuar negativas y el modo en la que éstas ocurren en las organizaciones. Por otro lado, de nuevo se observa una distinción entre las acciones que son hacia la organización y las que son para quienes trabajan dentro de ella. Como se ha mencionado, esta división un poco recurrente en cada uno de estos términos utilizados para abordar dicho fenómeno.

La postura de los comportamientos desviados en el trabajo y el comportamiento antisocial en las organizaciones tienen un punto en común, ya que ambas ponen su foco de interés en las distintas consecuencias que tienen para la organización y para quienes están dentro de ella. De esta manera, las clasificaciones que proponen los autores citados están construidas en función de a quién van dirigidas las acciones y en relación con cuáles son consideradas de mayor impacto.

En este análisis la propuesta que utiliza el término de comportamientos contraproducentes en el trabajo incluye una perspectiva más amplia, en el sentido de que refleja los factores que influyen en los comportamientos antisociales o desviados y una forma más clara de categorizar dichos factores. Este tipo de comportamientos se refieren de igual manera a los que ocurren de modo intencional y que son considerados por la organización como contrario a sus intereses legítimos, por lo que éstos siempre ocurren de manera voluntaria y afectan el desempeño de los individuos y de la organización (Lau, Au y Ho, 2003; Sacket y DeVore, 2001; Spector et al., 2006).

Sackett y DeVore (2001) argumentan que no deben entenderse como sinónimos los comportamientos contraproducentes en el trabajo y la contra productividad o la falta de productividad. Para dichos autores la segunda es una consecuencia de la primera, es decir, esta postura está marcada por la perspectiva de la organización y destaca el desempeño laboral. De modo que la ocurrencia de estos comportamientos impide que los miembros de las organizaciones logren los resultados esperados en el trabajo. De manera contraria a lo propuesto por Robinson y Bennet (1995), des- de esta perspectiva tiene un papel importante todo lo que afecte resultados o lo esperado por la organización.

Debido a la importancia que tienen estos comportamientos para los intereses de las organizaciones, prevalece la urgencia de una explicación más clara de los antecedentes o factores que propician estas acciones. Sackett y DeVore (2001) indican que las acciones negativas se deben sobre todo a las características de personalidad de los sujetos. Otras categorías consideran aspectos como antecedentes al desencadenamiento de estos comportamientos, las características del trabajo, las características del grupo de trabajo, la cultura organizacional y la percepción de la injusticia. Del mismo modo que los estudios referentes a comportamientos antisociales en la organización, Sackett y DeVore (2001) consideran que en esta postura se agrupan acciones negativas, como el robo, la destrucción de propiedad, el mal uso de información o del tiempo de trabajo y el uso de drogas, entre otros comportamientos que representan acciones de violencia hacia otros o que involucran un mal desempeño laboral (en contra de lo establecido por la organización).

Una última propuesta conceptual es la de Vardi y Weitz (2003), quienes introducen el término de mala conducta en la organización, definiéndolo como "los actos en el lugar de trabajo que se hacen intencionalmente y constituyen una violación de las reglas que se proporcionan a dichas conductas" (p. 3). Esta propuesta destaca la necesidad de abordar la problemática no sólo de modo individual o micro, sino de manera más extensa, estudiándola desde la relación que tienen los contextos organizacionales con las características individuales. En el mismo sentido, estos autores analizan las relaciones que establecen los miembros dentro de la organización manteniendo una postura macro de la problemática, pues considera elementos de la cultura organizacional y el ambiente.

La manera de nombrar a las acciones antisociales propuestas por Vardi y Weitz (2003) considera aspectos del contexto y del grupo social, al igual que la perspectiva de Robinson y O'Leary-Kelly (1998). Lo anterior refleja la importancia de los aspectos relacionados con el clima y la cultura organizacional referidos por Giacalone y Greenberg (1997). Por último, retoman los distintos factores que pueden propiciar estas acciones antisociales mencionadas por Sackett y DeVore (2001), quienes plantean categorías que reflejan los aspectos individuales en relación con los del mismo contexto organizacional.

Para Vardi y Weitz (2003) la perspectiva es igual a las mencionadas. Dan mayor relevancia al punto de vista de la organización, es decir, que ésta es la dicta las reglas como pauta para establecer lo que es una mala 
conducta o una conducta antisocial. Por otra parte, la postura del individuo como principal ejecutor de las acciones negativas hacia la organización se mantiene por las propuestas conceptuales comentadas. Al presentar algunos rasgos de personalidad en combinación con estímulos dentro del ambiente laboral se considera que llega a involucrarse en estas conductas.

A partir de los términos expuestos se da cuenta de los trabajos que buscan sobre todo el establecimiento de la relación entre las características de los individuos para explicar su influencia en los comportamientos antisociales. Hoy es posible encontrar aportaciones de Bowling y Eschleman (2010), quienes contribuyen al estudio de esta problemática indicando cómo los rasgos de personalidad tienen un efecto moderador en la relación del estrés y la manifestación de comportamientos contraproducentes en el trabajo, es decir, que los sujetos que presentan determinadas características de personalidad, como bajos niveles de conciencia y altos niveles afectividad negativa, serán más propensos a encaminar el estrés presentado en el trabajo hacia comportamientos negativos. Otros estudios también se enfocan en la inteligencia emocional o en la influencia de las emociones en la presencia de comportamientos contraproducentes. En este sentido, Ugwua, Enwereuzora, Fimbera y Ugwub (2017) muestran cómo es que la inteligencia emocional tiene un efecto moderador en la relación positiva entre la presencia del síndrome de burnout en trabajadores y las respuestas en forma de comportamientos contraproducentes. De manera que los trabajadores que presenten dicho síndrome, y a su vez mantengan un buen manejo de su inteligencia emocional, les ayudará a no inclinarse por acciones contraproducente en el trabajo.

Rahman, Ferdausy y Karan (2012) también indican cómo los individuos que presentan una menor inteligencia emocional son más propensos a involucrarse en comportamientos contraproducentes y a presentar peor desempeño laboral, es decir, que hay una relación negativa entre ambas variables; a menor grado de inteligencia emocional los empleados tienden a involucrarse más en estas prácticas en el trabajo. De acuerdo con los trabajos que se enfocan en aspectos emocionales, Shkoler y Tziner, (2017) analizan cómo la inteligencia emocional puede ser una característica de los individuos que logran contrarrestar la ocurrencia de comportamientos desviados cuando son influenciados por el estrés presentado en los miembros de una organización y por la percepción de justicia organizacional. Con estos resultados se puede entender que los sujetos con un nivel de inteligencia emocional adecuada pdrán manejar de mejor manera el estrés y evitar que se vean involucrados en actos que perjudiquen a otros o a la organización.
En esta misma línea, hay investigaciones que muestran la relación entre la percepción de falta de justicia en el contexto organizacional y el efecto mediador que tienen emociones negativas como el enojo y el miedo para que a partir de esto desencadenen en comportamientos contraproducentes (Le Roy, Bastounis y Minibas, 2012; Nisar, Bashir, Fatima y Ali, 2018).

En dichos estudios, como determinante que influye en la problemática en cuestión, existen trabajos que, a partir de los cinco grandes rasgos de personalidad, muestran cómo los sujetos con niveles altos en los rasgos de neuroticismo y los que presentan índices elevados en la escala de apertura a la experiencia, se ven más implicados en comportamientos contraproducentes en el lugar de trabajo (Firdaus, Safin, y Rahim, 2013; Ramana, Sambasivan y Kumar, 2016).

Por otra parte, en las investigaciones referentes a los comportamientos antisociales en las organizaciones también hay las que tienen en cuenta las condiciones laborales y los aspectos relacionados con el ambiente laboral como desencadenantes de dichos comportamientos, en relación con los aspectos individuales y rasgos de personalidad ya mencionados.

Es posible encontrar trabajos que establecen una relación entre la ocurrencia de los comportamientos contraproducentes, el estrés y la satisfacción laboral presentados en los sujetos de una organización (Aldea, 2013; Omar, 2010), es decir, una relación positiva entre estrés laboral y comportamientos contraproducentes y una relación negativa entre satisfacción laboral con dichos comportamientos. Los sujetos que cursen por situaciones de estrés laboral aumentarán la posibilidad de expresar comportamientos contraproducentes, y por otro lado los que experimentan menor satisfacción laboral dentro de la organización pueden verse más involucrados en los mismos. Siguiendo esta línea, Spector y Zhou (2013) indican la relación entre el estrés laboral y los comportamientos contraproducentes como un antecedente a estos comportamientos. Por otra parte, establecen que también los mismos comportamientos contraproducentes pueden ser los causantes del estrés laboral manifestado por los sujetos de una organización.

Zoghbi y Caamaño (2010) hacen aportaciones relevantes al considerar la alienación laboral como un factor que desencadena comportamientos desviados en el trabajo dirigido contra la organización, los compañeros o los clientes. Zoghbi y Caamaño (2010) definen la alienación laboral como la discrepancia o alejamiento de la persona de sus propios valores o de sí mismo, por consecuencia de las exigencias a que son expuestos en el trabajo, es decir, una contradicción entre cómo es el trabajador y la na- 
turaleza del trabajo. Al ocurrir esta situación el trabajador puede no encontrar un significado a sus tareas laborales, y es aquí donde se establece la relación con la ejecución de comportamientos contraproducentes.

En este sentido, Zoghbi y Caamaño (2010) indican que el comportamiento contraproducente de los individuos se establece a partir del constante intercambio de beneficios obtenidos por otros dentro del contexto organizacional y su relación con las tres dimensiones de la alineación laboral, las cuales abarcan la ausencia de control o impotencia en su trabajo desempeñado, y ausencia del significado en su trabajo desempeñado, es decir, sentir que su trabajo no genera influencia en el proceso de producción de la organización. Por último, la falta de sentimientos de realización a partir del trabajo desempeñado como un predictor de los comportamientos contraproducentes. La primera dimensión en relación con las acciones hacia la organización, la segunda hacia los compañeros y la tercera hacia los clientes. Los resultados muestran cómo las dos últimas tienen influencias significativas en los comportamientos contraproducentes de manera contraria a la primera.

Otra de las condiciones que se ha estudiado como antecedente es la percepción de supervisión abusiva. De esta manera, hay investigaciones que se refieren a la influencia de esta manera de supervisión, y cómo el afecto negativo presentado en el trabajo por parte de los individuos es un mediador entre la supervisión abusiva y la ejecución de comportamientos desviados en el trabajo (Eschleman, Bowling, Michel y Burns, 2014; Sulea, Fine, Fischmann, Sava y Dumitru, 2013; Michel, Newness y Duniewicz, 2016). A estos resultados le acompaña que los sujetos con puntuaciones bajas en rasgos de personalidad, como la estabilidad emocional, consciencia y afabilidad, se verán más inclinados a ser partícipes de comportamientos desviados al momento de ser sometidos a condiciones de supervisión abusiva. A partir de estos trabajos se ha buscado establecer a la supervisión abusiva como un predictor de los comportamientos negativos en los sujetos.

Respecto a las condiciones laborales o interacciones que se establecen entre trabajadores y los sujetos que desempeñan funciones de encargados, es posible encontrar los que hablan de la percepción de justicia en la organización (Syaebani y Sobri, 2011; Priesemuth, Arnaud y Schminke, 2013), donde la percepción de justicia organizacional es un claro predictor acerca de si ocurrirán comportamientos desviados en la organización por parte de los individuos. En esta misma línea, diversos autores (Chernyak y Tziner 2014; Kelloway, Francis, Prosser y Cameron, 2010) también destacan la percepción de justicia distributiva organizativa experimentada de manera psicológica y el clima organizacional como predictores del comportamiento contraproducente en el trabajo, sobre todo en las relaciones establecidas entre los miembros de menor hacia los que ocupan un puesto más alto en la organización.

Para dichos estudios los comportamientos antisociales pueden ser una respuesta de protesta donde los miembros de la organización expresan insatisfacción o intento de resolver la injusticia dentro de la organización. Es decir, los miembros de la organización que presentan mayor identificación con quienes persiguen intereses contrarios se verán más implicados en los comportamientos en contra de la organización con el objetivo de mitigar las condiciones mencionadas, así como aquellos en que no exista una percepción de encontrar beneficios dentro de su interacción con la organización.

Las investigaciones mencionadas en esta sección, bajo la postura de comportamientos contraproducentes en el trabajo, comportamientos desviados en el lugar de trabajo y mala conducta en el lugar de trabajo, destaca con más claridad la perspectiva de la organización y la importancia de clasificar como negativo todo lo que se perciba o se considere contradictorio para los objetivos que se pretendan lograr en dichos contextos.

Por otro lado, es evidente una tendencia a estudiar el fenómeno desde una perspectiva que considera características individuales como los rasgos de personalidad, pues son los trabajadores quienes sobre todo cometen estas acciones en contra de la organización. No obstante, desde esta perspectiva también son considerados aspectos del contexto de trabajo, siendo constante la dirección de cómo ocurren estos comportamientos trabajador hacia la organización. En estas últimas posturas, el desempeño laboral y los daños hacia la organización reflejan mayor importancia porque son las que padecen las consecuencias negativas de los comportamientos contraproducentes; esto representa un obstáculo para que las organizaciones logren sus objetivos.

En todas las formas de nombrar a esta problemática de estudio siempre se hace referencia a los comportamientos motivados por un empleado, o un grupo de empleados, con consecuencias negativas para otro individuo, grupo o la organización, lo que es constante en las definiciones y posturas de los autores citados. Los trabajos mencionados coinciden en que siempre habrá una pauta de normas y reglas establecidas por las organizaciones que determinarán quién ejecuta las acciones consideradas antisociales. 
disciplina. Al ser un problema dentro de estos contextos hay una gran diversidad de autores que no optan por este término, o han considerado más útil nombrarlo de otras maneras que otorgan mayor relevancia a la postura de la organización y a los daños económicos o productividad, en lugar de los otros daños mencionados como subjetivos, en donde se encuentran más los aspectos psicológicos y emocionales de los miembros.

Por tanto, muchas de las posturas propuestas por otros autores que han abonado al estudio de este problema, se enfocan en reflejar sobre todo esta importancia en común, es decir, el principal interés de los autores está dirigido en la comprensión de por qué ocurren y de las consecuencias que tienen para las organizaciones. En función de eso han clasificado su gravedad a partir de la postura de la misma organización. En caso de las acciones que han sido consideradas menos severas, llama la atención que éstas son aceptadas dentro de la dinámica del trabajo (como llegar tarde o los favoritismos).

En la postura de comportamientos desviados, contraproducentes y mala conducta organizacional hay una pauta similar. Todo lo que sea considerado en contra de la organización es tomado como una acción negativa o un comportamiento desviado en el trabajo. La pauta de lo que está bien o mal es marcado por la organización. Todo lo que se aleje o se considere perjudicial a los intereses legítimos o que impida el bienestar dentro del ambiente laboral para lograr los objetivos de la organización, se considera dentro de esta clasificación de comportamientos negativos.

Estas últimas posturas hacen referencia a cuando los miembros de las organizaciones o los trabajadores son quienes ejecutan las acciones negativas sin considerar, en su mayoría, otras interpretaciones que puedan darse en el contexto organizacional en relación con este mismo fenómeno por parte de los trabajadores. En este caso, el énfasis está más en la productividad o el desempeño laboral, en los resultados, en los aspectos y acciones contraproducentes en el trabajo.

Al estudiar este problema dentro de las organizaciones y analizar las posturas, es posible observar esa tendencia a una división entre la organización y sus miembros, como si se trataran de aspectos que hay que entender de manera separada. Es común que las explicaciones tiendan a ser en función de los rasgos de personalidad o características individuales de los miembros que, al ser expuestos a elementos del contexto de trabajo, propician acciones negativas hacia ella.

En relación con lo anterior es importante comenzar a rescatar las propuestas que hacen énfasis en iniciar el estudio de la problemática de manera social, porque las organizaciones son consideradas contextos sociales en donde hay una diversidad de grupos de personas e interacciones entre éstos. Es conveniente evitar la división entre la organización y sus miembros y comenzar a estudiar de modo integral el problema teniendo en cuenta las interacciones dentro de las organizaciones, las distintas interpretaciones, significados y sentidos que puede tener un mismo problema para cada uno de los grupos de están en un mismo contexto. Al considerar lo anterior, será posible obtener distintos elementos como los aspectos sociales, culturales, creencias, actitudes, estereotipos y opiniones que están detrás de un fenómeno complejo como el descrito en el este trabajo. Esto permitirá formas de intervención que puedan abarcar distintos elementos como los mencionados, y por tanto formas de intervención más integrales del problema.

\section{Conclusiones}

En este artículo se mostró un recorrido por los distintos términos o conceptos utilizados para el estudio de los comportamientos antisociales en las organizaciones. Se plasmó la prevalencia de la diversidad existente, y con ello es evidente que los autores se refieren al mismo problema de estudio. A partir de lo anterior también se mostraron las distintas formas de clasificación que han sido utilizadas. Del mismo modo, se ha expresado la manera en la que cada uno de los autores, de acuerdo con sus perspectivas, estudian la problemática en las organizaciones. Se logró reflejar cómo los comportamientos antisociales ya representan un problema que adquiere relevancia en estos contextos.

Al ser parte de las organizaciones, es un tema que resulta bastante complejo, pues se tienen que encontrar formas de prevención, por la diversidad de aspectos que comprende una organización. En este sentido es necesario unificar estos distintos términos y establecer una postura global para evitar confusiones en futuras investigaciones.

Por otra parte, debido a la complejidad del fenómeno se propone que debe ser estudiado considerando tanto los aspectos individuales como los del grupo social, así como todas las características de las organizaciones en relación con el ambiente laboral, porque esta problemática no puede ser sólo estudiada desde una postura individual, teniendo en cuenta que las organizaciones son contextos sociales en los cuales las personas interactúan de modo constante. Se debe dejar de lado la perspectiva de la organización, así como la división entre el trabajador y la organización, esto con el objetivo de evitar limitaciones para futuros trabajos, permitiendo así desarrollar formas de investigación e intervención más integradas.

En cuanto a las limitaciones de este trabajo, no se muestra una revisión sistemática de los antecedentes por- 
que el objetivo fue la clarificación conceptual y mostrar las distintas posturas que guarda el uso de un concepto sobre otro para estudiar un mismo problema. Sin embargo, se reconoce que es pertinente hacer también la revisión mencionada, lo cual puede contribuir a plasmar la tendencia acerca del abordaje actual del problema. Con los estudios mostrados hasta el momento puede inferirse la inclinación más hacia el trabajo cuantitativo, buscando correlaciones y relaciones causales en torno a los factores que pueden propiciar este tipo de comportamientos en las organizaciones, por lo que han quedado de lado los aspectos subjetivos que pueden estar detrás de la ocurrencia de estas acciones dentro de las organizaciones y las formas de interpretar un mismo fenómeno por cada grupo social que componen dichos contextos.

\section{Referencias}

Aldea, R. (2013) Antecedents and mediators of employees, counterproductive work behavior and intentios to quit. ProcediaSocial and Behavioral Sciences:An Exploratory Study. Procedia Economics and Finance, (7),181 - 187.

Appelbaum, S., Iaconi, G., \& Matousek, A. (2014). Positive and negative deviant workplace behaviors: causes, impacts and solutions. Corporate Governance International Journal of Business in Society, 7 (5), 586-598.

Bowling, N. A., \& Eschleman, K. J. (2010). Employee personality as a moderator of the relationships between work stressors and counterproductive work behavior. Journal of Occupational Health Psychology, 15 (1), 91-103.

Chernyak-Hai, L., \& Tziner, A. (2014). Relationships between counterproductive work behavior, perceived justice and climate, occupational status, and leader-member exchange. Revista de Psicología del Trabajo y de las Organizaciones, 30 (1), 1-12.

Eschleman, K. J., Bowling, N. A., Michel, J. S., \& Burns, G. N. (2014). Perceived intent of supervisor as a moderator of the relationships between abusive supervision and counterproductive work behaviours. Work \& Stress, 28 (4), 362-375.

Firdaus, I. A., Safin, S. Z., \& Rahim, A. A. (2013). The relationship of big five personality traits on counterproductive work behaviour among hotel employees: An exploratory study. Procedia Economics and Finance (7), 181-187.

Giacalone, R., \& Greenberg J. (1997). Antisocial behavior in organization. EUA: Sage.

Griffin, R. W., \& O'Leary-Kelly, A. (2004). The Dark Side of Organizational Behavior. New York: Pfeiffer/Jossey-Bass.

Griffin, R., \& Lopez, Y. (2005). "Bad Behavior" in Organizations: A Review and Typology for Future Research. Journal of Management (31), 988.

Kelloway, E., Francis, L., Prosser, M., \& Cameron, J. (2010). Counterproductive work behavior as protest. Human Resource Management Review (20), 18-25.

Lau, V., Au, W., \& Ho, J. (2003). A qualitative and quantitative review of antecedents of counterproductive behavior in organizations.
Journal of Business and Psychology (18), 73-100.

Le Roy, J., Bastounis, M., \& Minibas, J. (2012). Interactional Justice and Counterproductive Work Behaviors: The Mediating Role of Negative Emotions. Socialbehavior and Personality, Society for Personality Research, 40 (8), 1341-1355.

Michel, J. S., Newness, K., \& Duniewicz, K. (2016). How abusive supervision affects workplace deviance: A moderatedmediation examination of aggressiveness and workrelated negative affect. Journal Bussines Psychology (31), 1-22. doi: 10.1007/s10869-015-9400-2.

Nisar, M., Bashir, M., Fatima, T., \& Ali, S. (2018). The underlying mechanism between perceived organizational injustice and deviant workplace behaviors: Moderating role of personality traits. Asia Pacific Management Review, 30 (1), 11.

Omar, A. (2010) Comportamientos extra papel: de la colaboración a la transgresión. Revista Interamericana de Psicología Ocupacional Investigaciones y Análisis, 29 (1), 32-53.

Omar, A., Vaamonde, J., \& Uribe, H. (2012). Comportamientos contraproducentes en el trabajo: Diseño y validación de una escala. Divers. Perspect. Psicol, 8 (2), 249-265.

Patlán, J., García, M. D. R., \& Navarrete, D. M. (2011). El lado obscuro de las organizaciones y del comportamiento organizacional. Gest. Soc., 4 (2), 81-92.

Patlán, J., Navarrete, D. M., \& García, M. (2010). El lado oscuro de las organizaciones: Efectos negativos en el factor humano. Revista del Centro de Investigación. Universidad La Salle, 9 (34), 71-86.

Priesemuth, M., Arnaud, A., \& Schminke, M. (2013). Bad behavior in groups the impact of overall justice climate and functional dependence on counterproductive work. Group and Organization Management, 38 (2), 230-257.

Rahman, S., Ferdausy, S., \& Karan, R. (2012). Relationship among emotional intelligence, deviant workplace behavior and job performance: An empirical study. Portuguese Journal of Management Studies, 7 (1).

Ramana, P., Sambasivan, M., \& Kumar, N. (2016). Counterproductive work behavior among frontline government employees: Role of personality, emotional intelligence, affectivity, emotional labor and emotional exhaustion. Journal of Work and Organizational Psychology (32), 25-37.

Robinson, S. L., \& O'Leary-Kelly, A. M. (1998). Monkey see, monkey do. The Influence of Work Groups on the Antisocial Behavior of Employees. The Academy of Management Journal, 41 (6), 658-672.

Robinson, S., \& Bennett, R. (1995). A typology of deviant workplace behaviors: A multi-dimensional scaling study. Academy of Management Journal, 38, 555-572.

Sackett, P. R., \& DeVore, C. J. (2001). Counterprooductive behaviors at work. In N. Anderson, D. S. Ones, H. K. Sinangil \& C. Viswesvaran (eds.). Handbook of Industrial, Work, \& Organizational Psychology. Vol. 1 (pp. 145-164). London, UK: Sage.

Shkoler, O., \& Tziner, A. (2017). The mediating and moderating role of burnout and emotional intelligence in the relationship between organizational justiceand work misbehavior. Journal of Work and Organizational Psychology, 33, 157-164.

Spector, P., Fox, S., Penney, L., Bruursema, K., Goh, A., \& Kessler, 
S. (2006). The dimensionality of counterproductivity: ¿Are all counterproductive behaviors created equal?. Journal of Vocational Behavior, 68, 446-460.

Spector, P., \& Zhou, Z. (2013). The Moderating Role of Gender in Relationships of Stressors and Personality with Counterproductive Work Behavior. Journal of Business and Psychology (29), 669-681.

Sulea, C., Fine, S., Fischmann, G., Sava, F., \& Dumitru, C. (2013). Abusive supervision and counterproductive work behaviors the moderating effects of personality. Journal of Personnel Psychology, 12 (4), 196-200. doi: 10.1027/18665888/a000097.

Syaebani, M. I., \& Sobri, R. R. (2011). Relationship between organizational justice perception and engagement in deviant workplace behavior. The South East Asian Journal of Management, 5 (1).

Ugwua, L. I., Enwereuzora, I. K., Fimbera, U. S., \& Ugwub, D. I. (2017). Nurses' burnout and counterproductive work behavior in a Nigerian sample: The moderating role of emotional intelligence. International Journal of Africa Nursing Sciences (7), 106-113.

Vardi, Y., \& Weitz, E. (2003). Misbehaviors in organizations: A dynamic approach. USA: Routledge.

Zoghbi, P., \& Caamaño, G. (2010). El impacto de la alienación laboral sobre las conductas desviadas en el trabajo: Un estudio exploratorio. Revista de Psicología del Trabajo y de las Organizaciones (26), 79-92.

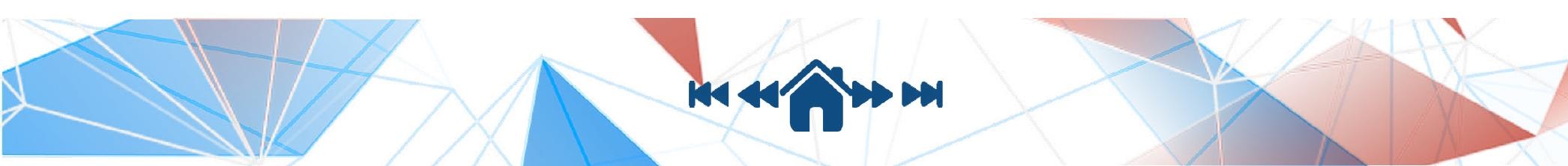




\section{Meta-Análisis del Artículo}




\section{Dimensión Cuantitativa}

\section{Perfil de Evaluación entre pares}
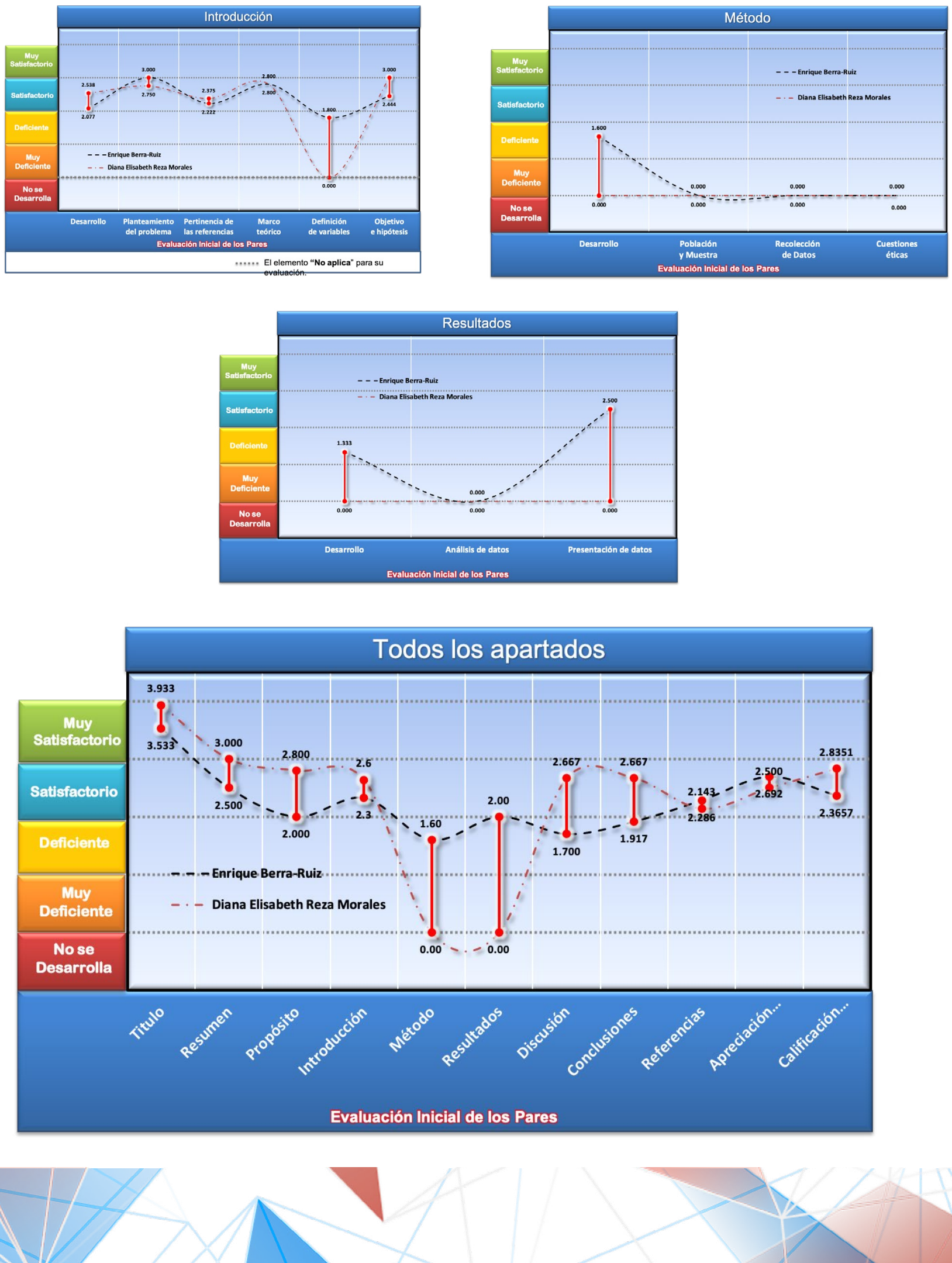


\section{Índice de Concordancia}

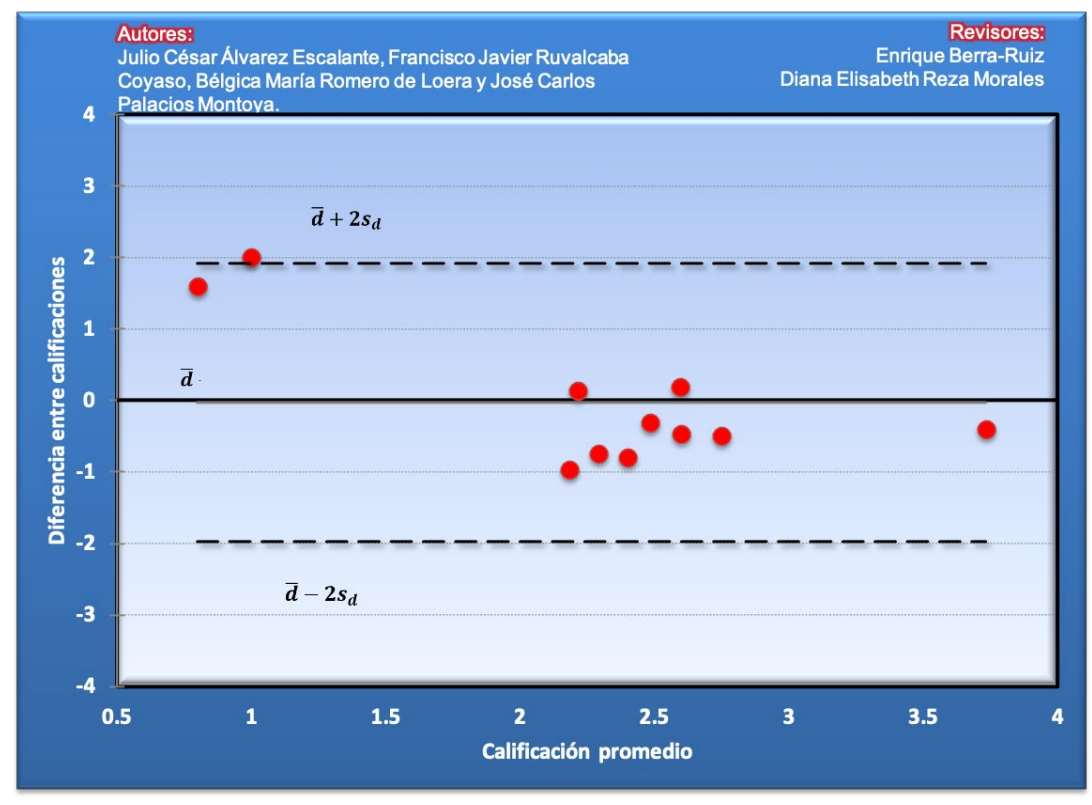

Índice de Acuerdo

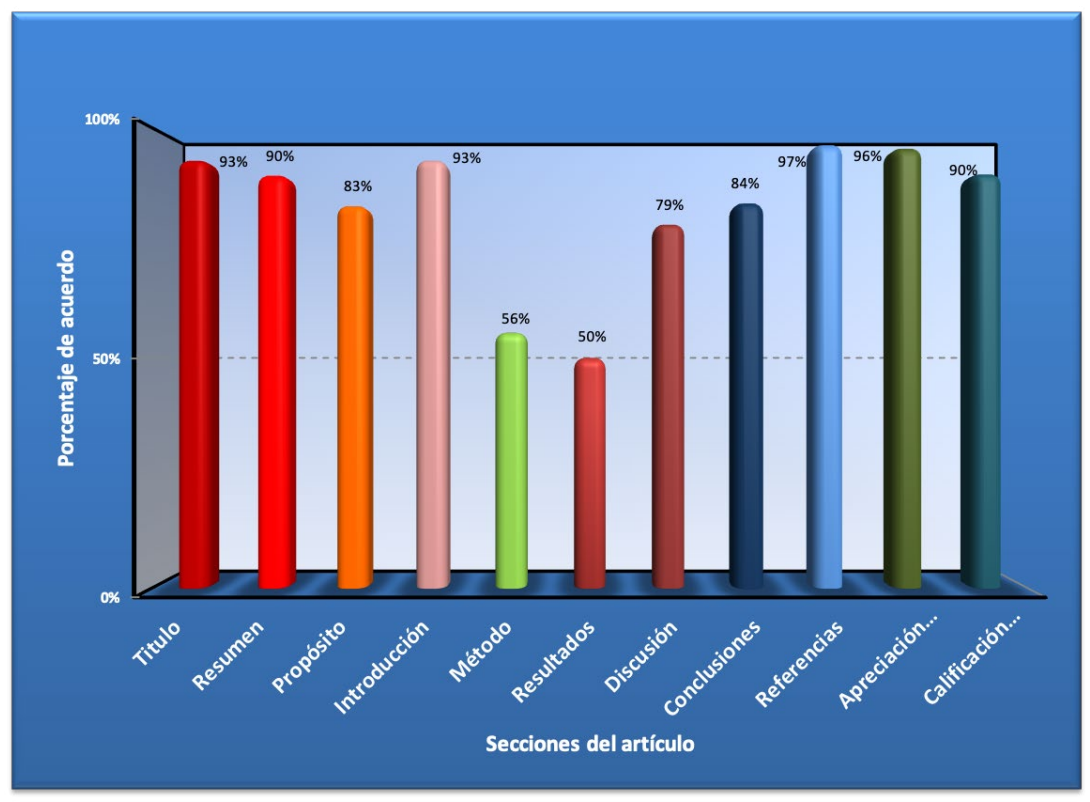




\begin{tabular}{|c|c|}
\hline Revisor 1 & Revisor 2 \\
\hline Enrique Berra Ruiz & Diana Elisabeth Reza Morales \\
\hline \multicolumn{2}{|c|}{ Título/Autoría } \\
\hline $\begin{array}{l}\text { El título da una idea clara de lo que se busca exponer en } \\
\text { el documento. Los datos de contacto se muestran única- } \\
\text { mente de un autor, se recomienda incluir los datos de to- } \\
\text { dos los autores. }\end{array}$ & En el título reemplazar; por: \\
\hline \multicolumn{2}{|c|}{ Resumen } \\
\hline $\begin{array}{l}\text { El resumen es menor a } 150 \text { palabras no integra todos } \\
\text { los elementos sobre todo en la sección del análisis, re- } \\
\text { sultados y conclusiones. }\end{array}$ & $\begin{array}{l}\text { 1)Se sugiere mejorar la redacción para que sea más clara la } \\
\text { conexión entre las ideas. 2) Se pueden ocupar sinónimos } \\
\text { para que las palabras no sean tan repetitivas. 3) Agregar } \\
\text { palabras clave. }\end{array}$ \\
\hline \multicolumn{2}{|c|}{ Próposito del Estudio } \\
\hline $\begin{array}{l}\text { El propósito es claro pero al paso de la lectura va desva- } \\
\text { neciendo el abordaje del problema. }\end{array}$ & $\begin{array}{l}\text { En el análisis conceptual que se propone dentro del tex- } \\
\text { to, hace falta citar a los autores, con la finalidad de brin- } \\
\text { dar un sustento sólido al argumento. }\end{array}$ \\
\hline \multicolumn{2}{|c|}{ Introducción } \\
\hline $\begin{array}{l}\text { El artículo requiere determinar mejor su marco teórico, } \\
\text { identificando mejor su abordaje de la problemática, se re- } \\
\text { quiere integrar mejor los objetivos específicos para brin- } \\
\text { dar una mayor congruencia entre las variables con la pro- } \\
\text { blemática delimitada. }\end{array}$ & $\begin{array}{l}\text { Para brindar solidez al trabajo se recomienda agregar más } \\
\text { referencias de autores que hablen de las conductas desvia- } \\
\text { das/antisociales/contraproducentes en las organizaciones, } \\
\text { complementando con estudios que den sustento a la vali- } \\
\text { dez de tales definiciones. }\end{array}$ \\
\hline \multicolumn{2}{|c|}{ Método } \\
\hline $\begin{array}{l}\text { Al ser un artículo teórico no aplicada gran mayoría de } \\
\text { estos elementos. }\end{array}$ & $\begin{array}{l}\text { Ningún comentario, ya que esta sección no aplica para un } \\
\text { artículo de revisión teórica. }\end{array}$ \\
\hline \multicolumn{2}{|c|}{ Resultados } \\
\hline $\begin{array}{l}\text { Al se un artículo teórico no aplican diversos rubros en su } \\
\text { valoración. }\end{array}$ & No aplica \\
\hline \multicolumn{2}{|c|}{ Discusión } \\
\hline $\begin{array}{l}\text { Se requiere refinar la discusión desde un abordaje más } \\
\text { claro en los antecedentes y desarrollo de la revisión teó- } \\
\text { rica que se plantea de la problemática. }\end{array}$ & $\begin{array}{l}\text { Agregar referencias para dar un sustento más sólido en el } \\
\text { apartado de análisis conceptual. }\end{array}$ \\
\hline
\end{tabular}


Artículo Teórico | Comportamiento antisocial en las organizaciones... Álvarez-Escalante, et al.

\section{Revisor 1}

Revisor 2

\section{Conclusiones}

Las conclusiones derivan una fracción del objetivo principal, quedando corto el abordaje inicial que se planteaba.

Se sugiere quitar del título la palabra revisión, ya que en las conclusiones se comenta que no se hizo una revisión del estado del arte de la temática, o bien especificar que tipo de revisión se hizo desde un inicio. Ampliar las conclusiones con lo que se encontró a nivel conceptual, no poner únicamente el recorrido que se hizo para efectuar la investigación.

\section{Referencias}

Para una revisión teórica son muy pocas las referencias empleadas, asimismo se requiere ajustar el listado de referencias bajo el mismo estilo.

1) Verificar las citas al formato APA 6ta edición 2) Verificar errores en la escritura del nombre de los autores. 3) Ampliar la cantidad de referencias de investigadores que hablan del tema a nivel conceptual y empírico. 


\section{Historia del Proceso EDITORIAL}

